\title{
Social VR: A New Medium for Remote Communication and Collaboration
}

\author{
Jie Li \\ jie.li@cwi.nl \\ Centrum Wiskunde \& Informatica \\ Amsterdam, NL
}

\author{
Vinoba Vinayagamoorthy \\ vinoba.vinayagamoorthy@bbc.co.uk \\ $\mathrm{BBC}$ R\&D \\ London, UK
}

\author{
Julie Williamson \\ Julie.Williamson@glasgow.ac.uk \\ University of Glasgow \\ Glasgow, UK
}

\author{
David A. Shamma \\ Rochester Institute of Technology \\ New York, US
}

Pablo Cesar

Centrum Wiskunde \& Informatica, Delft University of Technology

Amsterdam, NL

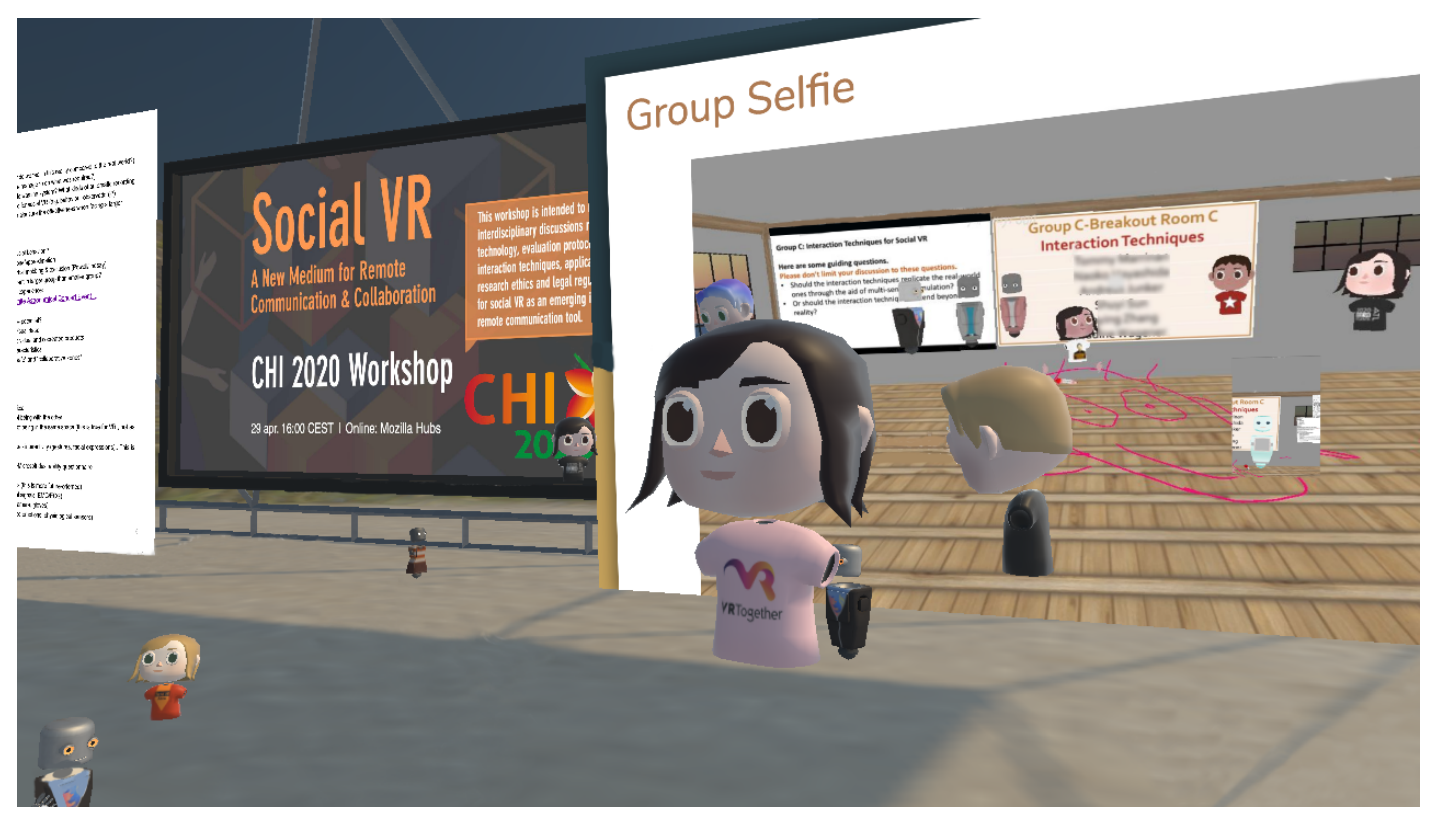

Figure 1: The CHI 2020 Social VR Workshop was hosted virtually on Mozilla Hubs.

\begin{abstract}
We are facing increasingly pressure on reducing travel and working remotely. Tools that support effective remote communication and collaboration are much needed. Social Virtual Reality (VR) is an emerging medium, which invites multiple users to join a collaborative virtual environment (VE) and has the potential to support remote communication in a natural and immersive way. We successfully organized a CHI 2020 Social VR workshop virtually on Mozilla Hubs, which invited researchers and practitioners to have a fruitful discussion over user representations and ethics, evaluation methods, and interaction techniques for social VR as an emerging

Permission to make digital or hard copies of part or all of this work for personal or classroom use is granted without fee provided that copies are not made or distributed for profit or commercial advantage and that copies bear this notice and the full citation on the first page. Copyrights for third-party components of this work must be honored. For all other uses, contact the owner/author(s).

CHI '21 Extended Abstracts, May 8-13, 2021, Yokohama, Japan

(c) 2021 Copyright held by the owner/author(s).

ACM ISBN 978-1-4503-8095-9/21/05.

https://doi.org/10.1145/3411763.3441346
\end{abstract}

immersive remote communication tool. In this CHI 2021 virtual workshop, we would like to organize it again on Mozilla Hubs, continuing the discussion about proxemics, social cues and VE designs, which were identified as important aspects for social VR communication in our CHI 2020 workshop.

\section{CCS CONCEPTS}

- Human-centered computing $\rightarrow$ HCI design and evaluation methods; Virtual reality; Collaborative interaction.

\section{KEYWORDS}

Social virtual reality, proxemics, remote communication, virtual environment design, social cues 
ACM Reference Format:

Jie Li, Vinoba Vinayagamoorthy, Julie Williamson, David A. Shamma, and Pablo Cesar. 2021. Social VR: A New Medium for Remote Communication and Collaboration. In CHI Conference on Human Factors in Computing Systems Extended Abstracts (CHI '21 Extended Abstracts), May 813, 2021, Yokohama, Japan. ACM, New York, NY, USA, 6 pages. https: //doi.org/10.1145/3411763.3441346

\section{INTRODUCTION}

As a continuation of the CHI 2020 social VR workshop [13], this workshop is intended to follow up the interdisciplinary discussions on social VR, with a focus on understanding the proxemics, social cues and virtual environment (VE) designs for social VR as an immersive remote communication tool. We are facing increasingly pressure on reducing travel and collaborating remotely. There is a growing need for effective remote communication, which has many positive societal impacts, such as reducing environmental pollution and travel costs, supporting rich collaboration by remotely connecting talented people. Video conferencing tools, such as Zoom ${ }^{1}$ and Google Hangouts ${ }^{2}$, are low-cost, allow multiple users to have conversations at the same time, and provide face-to-face-like experiences compared to audio-only phone calls $[8,12]$. Some high-end video conferencing systems such as HP Halo and Cisco Telepresence are designed to link two physically separated rooms through wallsize screens, high-fidelity audio and video, which enable users to feel co-present in a single conference room $[2,23]$. However, all the video conferencing tools still restrict users in front of screens with "talking heads experiences", and limit physical activities that naturally arise from social interactions and spontaneous collaborations $[7,12]$.

Social VR has the potential to afford more social interaction than video conferencing, such as the ability to organically break off into small groups, or interacting with virtual objects in the scene [19]. Many commercial social VR platforms have implemented novel social mechanics to stimulate social activities, such as designing a VE to simulate group discussion atmosphere, implementing built-in tools to enable users to stay in VEs and focus on the social tasks. Existing social VR platforms vary widely in affordances, fidelity, scale, and accessibility. On commercial platforms such as Facebook Horizon $^{3}$, AlterSpaceVR ${ }^{4}$, and VRChat ${ }^{5}$, the facial expressions, voice, eye direction and body gestures of a user are captured and mapped to the virtual avatar of that user in real time. Platforms like Mozilla Hubs, Gather Town ${ }^{6}$ also enable social experiences, but result in dramatically different experiences. Facebook Horizon require users to have a head-mounted display (HMD). AlterSpaceVR, VRChat and Mozilla Hubs provide fully 3D environments that can be experienced on a desktop or using an HMD. Gather Town uses a 2D map, but incorporates video conferencing for groups to chat. AlterSpaceVR and VRChat are massively online VR community,

\footnotetext{
${ }^{1}$ Zoom is a video conferencing tool, enabling a large group of people meeting online at the same time.

${ }^{2}$ Google Hangouts is a multiple-user video conferencing tool.

${ }^{3}$ Facebook Horizon is an invite-only virtual community where users can explore the virtual worlds and do creative activities together.

${ }^{4}$ AlterSpaceVR is a commercial virtual reality community for virtual live shows, meetups, classes.

${ }^{5}$ VRChat is an online massively multiplayer social environment.

${ }^{6}$ Gather Town combines 2D maps with video conferencing.
}

averaging over 10,000 users daily. In contrast, Mozilla Hubs and Gather Town support a maximum of 25 and 50 users respectively, although premium Gather Town rooms can host up to 500. Facebook Horizon, AlterSpaceVR, and VRChat require a relatively high specification PC suitable for gaming, but Mozilla Hubs and Gather Town run in most standard browsers. Accessibility in VEs presents an on-going challenge [24], and open source social VR platforms like Mozilla Hubs create the best opportunities to address accessibility requirements. This is especially important for scholarly community events which must prioritise accessibility and inclusion. This is the reason why we chose Mozilla Hubs to run the CHI 2020 social VR workshop.

All these platforms have shown that social VR becomes a promising new medium for remote communication, which may better support social presence (e.g., intimacy and immediacy [17]), rich non-verbal communications (e.g., sign languages [25]), and immersive realistic interactions. However, the goal of social VR is not to completely replicate reality, but to facilitate and extend existing communication channels of the physical world. Besides, we are aware of the ethical risks of social VR systems. While human representations in VR become increasingly realistic, and research on HMD removal (e.g., [27]) is trying to make user faces visible, privacy concerns are raised (e.g., [18]). In our previous workshop, we extensively discussed three main topics: (1) user representation realism and its impact on privacy and ethics, (2) evaluation methods and protocols for understanding social VR experiences, and (3) new interaction techniques dedicated to enhance social VR communication. In this CHI 2021 new workshop [13], we intend to spur discussions over three other topics which were identified as crucial for natural and immersive social VR communication and collaboration, namely proxemics, social cues and VE designs.

\section{BACKGROUND}

This section presents the background work related to proxemics, social cues and VE designs.

\subsection{Proxemics and personal space}

The dynamics in interpersonal distances is an integral part of nonverbal communication in a shared space, which adds to the richness and versatility of the conversation [3]. Hall's theoretical model of proxemics introduced four concentric zones to define different levels of social intimacy, namely intimate $(<0.45 \mathrm{~m})$, personal $(0.45 \mathrm{~m}-$ $1.2 \mathrm{~m})$, social $(1.2 \mathrm{~m}-3.6 \mathrm{~m})$ and public $(>3.6 \mathrm{~m})$ [9]. However, there are many factors which affect the proximal relationship between people including age, culture, environmental context, interpersonal relationship and emotional state of the individuals [3, 5, 10, 20, 22]. Proximal behaviour is also interconnected with other modalities of expression such as gaze behaviour. Argyle and Dean's [4] theory of equilibrium posits that appropriate interpersonal distance is maintained between individuals by balancing increasing proximity with reduced eye contact. The lower limits of the distance is determined by physical contact whereas the upper limit is defined by factor of visibility and audibility [9].

"Personal space" is clearly important in both physical and virtual worlds. However, we don't have a complete picture of proxemics and personal space when applied to virtual spaces. Hecht et al. [11] 
reported that the shape of personal space in the real world was remarkably close to a circular zone with a radius of about one meter and this was similar to the personal distance participants maintained to an avatar in a virtual space. Yee et al. [26] conducted a observational study in Second Life and found that social norms of gender, interpersonal distance and gaze behaviour transferred to VEs. They found support for the equilibrium theory through avoidance of collision. In addition, the results showed that male dyads maintained larger interpersonal distances and less mutual gaze than female dyads. In a similar vein, Bonsch et al. [6] reported that participants chose to maintain larger interpersonal distances to virtual agents perceived to be angry in comparison to happy virtual agents. Recently, McVeigh-Schultz et al. [14] laid out some design considerations that might shape social interactions in shared virtual spaces including aesthetics of VEs, embodied affordances, social mechanics, and tactics for shaping social norms and mitigating harassment.

\subsection{The design of virtual space and social cues}

Virtual Environments (VEs) are often crafted as representations of physical spaces with the aim to facilitate a virtual event. As with physical environments, the design of VEs influences how people form groups and join activities. Inspired by urban ethnography, Moore et al. describe the environment design in terms of accessibility, social density, activity resources, and hosts [15]. For example, large open spaces can be difficult to fill with enough people to achieve "social density". Virtual spaces that are not restricted by physical building or space constraints often fail to create cosy places where social contact comes easily.

Moving from the physical world to VEs may change social activities: creating new forms of interaction (e.g., use controllers to teleport in VEs), and new social norms to keep the virtual world in order [16]. Ackerman et al. [1] studied social regulation in an online game. They show specific social norms, defined to regulate the game world interactions and technical interventions, are imposed in game to automatically prevent or punish unwanted behaviours. Yee et al. [26] indicated that social interactions in VEs are governed by the same social norms (i.e., social norms of gender, interpersonal distance, and eye gaze) as social interactions in the physical world. Social VR has the potential to afford more social interaction than video conferencing, such as the ability to organically break off into small groups, or interacting with virtual objects in the scene [19] Many commercial social VR platforms have implemented novel social mechanics to stimulate social activities, such as designing a VE to simulate group discussion atmosphere, implementing built-in tools to enable users to stay in VEs and focus on the social tasks, or enabling users to use simple hand gestures to stop harassment [14].

\section{PARTICIPANTS AND EXPECTED INTERESTS}

Social VR has not only attracted attention from academia, but also from commercial companies, such as AltspaceVR, Sansar and Facebook Horizon, all of which seek to include social features in their systems [21]. Therefore, we welcome all fields of interest: computer scientists, developers, artists, psychologists, HCI researchers, UX designers, and governmental policymakers etc., to jointly discuss topics about proxemics, social cues, VE designs, and explore the new use scenarios of social VR. We expect participants of diverse expertise will have interdisciplinary discussions on social VR topics, resulting in multifaceted new research agenda towards the future of social VR.

\section{WORKSHOP PLANS AND SCHEDULE}

The workshop is planned to be a 4-hour virtual event that will be held on a social VR platform called Mozilla Hubs, which is accessible both on web browsers on phones, tablets and desktop computers, and on head mounted displays (HMDs). The virtual workshop space will be designed to resemble a physical conference hall, with big screens to show the presentation slides, and open space for people to meet and talk. We will also build three virtual breakout rooms for participants to have separate group discussions (Figure 2).

A pre-workshop survey will be handed out to participants to order their preferences over the three topics, namely proxemics, social cues and VE designs. According to their preferences, the participants will be evenly assigned to discussion groups before the workshop starts. All participants will be requested to make 1-3 slides in a PDF format about their submitted position papers. The font size in the slides should not be smaller than 30 points. The organizers will combine all the PDF slides and upload them to the virtual workshop space in advance.

Due to the limitation of the platform, we will only invite 2025 participants. One keynote talk will be given at the beginning about organizing events and conducting research on social VR platforms. After the keynote, the participants will be asked to give a 2-minute pitch about their position papers. Then, they will find their pre-defined discussion groups, join the breakout room and focus on group work and discussion. Every discussion group will be facilitated by one workshop organizer.

We have successfully run our CHI 2020 social VR workshop on Mozilla hubs. For this workshop, we will instrument Mozilla Hubs with accessible features for captioning and navigation (e.g., participants can attend the workshop on Mozilla Hubs using desktop computers, mobile phones and HMDs). Table 1 presents the tentative schedule of the workshop. To welcome participants from different time zones, we plan to run the workshop from 16:00 to 20:00 at the time zone of the Central European Summer Time. We will distribute a Call for Participation (CFP) to relevant research institutes and universities and on social media. We will also send invitations to potential researchers and practitioners. Submitted position papers will be reviewed and selected by the workshop organizers. Our website will act as portal to advertise the workshop, and to inform and keep contact with the accepted participants.

\section{WEBSITE}

The Home page of the website displays the goal, important dates of the workshop and a button to submit position paper. The Call for Participation page describes the main topics of the workshop. The Organizers and Contact pages display the profile photos, contact emails, and personal websites of the organizers. The website is located at https://www.socialvr-ws.com. 


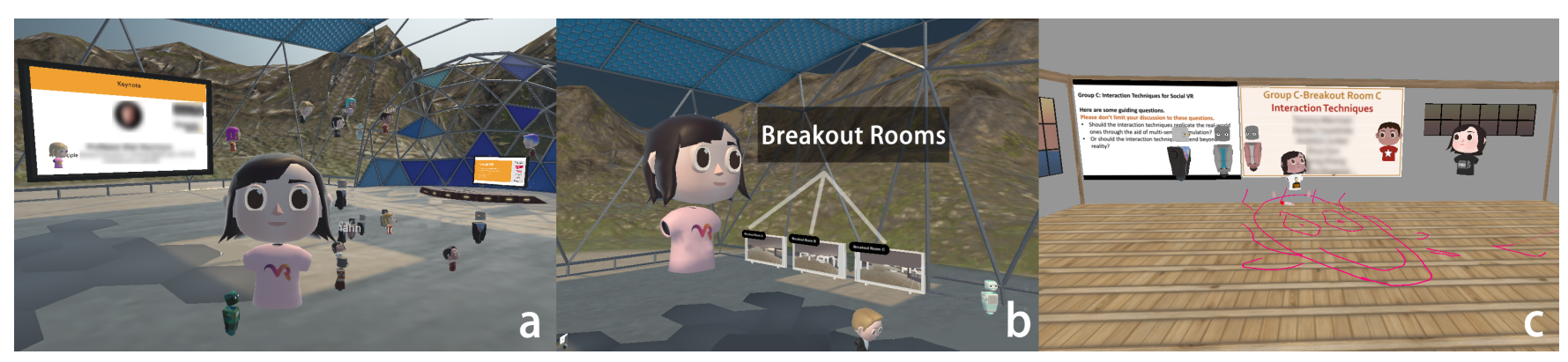

Figure 2: The virtual space design of the workshop on Mozilla Hubs: (a) The main workshop hall for the keynote talk and the pitches; (b) The three links in the main hall, directing participants to the virtual breakout rooms; (c) One of the virtual breakout rooms. Participants are represented as avatars and communicate with each other using audio.

Table 1: Tentative workshop schedule based on the previous successful run of our 2020 schedule.

\begin{tabular}{ll}
\hline Time & Activities \\
\hline May 6, 2021 & \\
16:00-17:00 & Training: Train participants to use Mozilla Hubs. \\
\hline May 8/9, 2021 & \\
15:45-16:10 & Warm-up: Login the virtual workshop and meet all the participants. \\
16:10-16:20 & Welcome: Introduce organizers, participants, workshop objectives and schedules. \\
16:20-16:50 & Keynote: Social VR as a new medium for remote communication \& collaboration. \\
16:50-17:00 & Q \& A \\
17:00-17:10 & Break \\
\hline 17:10-17:40 & Pitches: Each participant gives a 2-min pitch about the position paper. \\
17:40-17:55 & Break \\
\hline 17:55-18:05 & Join discussion groups: Join the pre-defined discussion groups in the breakout rooms. \\
18:05-18:45 & Group Discussion: Every discussion group will be facilitated by one organizer. \\
18:45-19:00 & Break \\
\hline 19:00-19:20 & Summarize discussion: Each group makes 1-3 slides to summarize the discussion results. \\
19:20-19:30 & Break \\
19:30-19:45 & Group presentation: Each group gives a 5-min presentation of the discussion results. \\
19:45-20:00 & Wrap up: Summarize the workshop and take virtual group photos. \\
\hline
\end{tabular}

\section{CALL FOR PARTICIPATION}

This section presents the "call for participation" that is going to be published on the workshop website.

\section{Social VR: A New Medium for Remote Communication and Collaboration}

Remote communication allows people who are not physically present in the same location to communicate with each other in real-time. This permits us to meet colleagues overseas, work from home to reduce commute cost and live far from our friends and families. Social Virtual Reality (VR) invites multiple users to join a collaborative virtual environment (VE), which creates new opportunities for remote communication. The shared experiences obtained in social VR may reshape our subjective perception towards the physical world, leading to shifts in our understanding about social experience, personal spaces, or realness of the VE, and bringing about novel everyday social interactions. However, social VR also raises privacy concerns and ethical risks when the boundary between the real and the virtual world is blurred. In this workshop, we intend to spur discussions on social VR as an emerging immersive remote communication tool.

The exciting news is that this social VR themed workshop will be held virtually on a social VR platform called Mozilla Hubs. We would like invite academics from all fields, e.g., computer science, psychology, HCI/UX, sociology, and designers, developers, practitioners, governmental policymakers to gather together on a social VR platform and to envision a future research agenda for social VR in terms of proxemics, social cues and VE designs.

\section{Important Dates}

Submission Deadline: February 21st, 2021

Notification: February 28th, 2021 
This virtual workshop invites submission of position papers: 2-4 pages in SIGCHI Extend Abstract format (reference excluded) via https://www.socialvr-ws.com, covering (but not limited to) the following topics:

\section{Topics}

- Personal Space in Social VR. How interpersonal distance is perceived in social VR when people can move in VEs beyond reality (e.g., flying)? What may be the differences in users' personal space perception when they using a desktop compared to wearing an HMD? What is the ideal interpersonal distance in VR collaboration?

- Social Cues. What are the missing social cues in existing social VR platforms? How do users start a conversation in a VE? What are the most necessary social cues to ensure natural and immersive collaboration, eye contacts, hand gestures, head movements?

- VE Designs. How to design the VEs to better facilitate virtual collaboration? How to design the VEs to prevent harmful virtual behavior? How to design VEs to ensure that it is aesthetically appealing and functionally sound (not distracting, and helping users focus on the virtual tasks)?

- Social VR Technologies. What is the current status of technology (e.g. capturing, reconstruction, rendering)? What are the technological requirements for improving social VR experiences in terms of quality of interaction, privacy protection etc.?

- Evaluation Protocols for Social VR Experiences. How to adequately evaluate different aspects of communication in social VR both subjectively (e.g., self-reports) and objectively (e.g., physiological sensors)? How to develop Quality of Experience (QoE) metrics for social VR?

- Interaction Techniques for Social VR. Should the interaction techniques replicate the real-world ones through the aid of multi-sensory simulation? Or should the interaction techniques extend beyond the reality?

Submitted position papers will be reviewed and selected by the workshop organizers. At least one author of the accepted paper must attend the workshop. All participants must register for both the workshop and for at least one day of the conference.

\section{EXPECTED OUTCOMES AND POST-WORKSHOP PLAN}

Through this virtual workshop, we expect to connect a community of researchers, commercial companies and artists interested in social VR technology, to further shape the technology, explore new use scenarios, initiate research collaborations and new project proposals on social VR topics. With consent from participants, we will collect activity data on the social VR platform (e.g., position, face direction, movement trajectory), player interaction status, and instrument further collection around about our proposed topics to provide an overview of benefits, challenges, and risks of using social VR as a new communication tool. The collected data will enable us to further understand research related to proxemics, social cues and virtual environment designs. We also plan on this data being open and scrubbed of personal identifying information.

\section{ORGANIZERS}

Jie Li is a postdoctoral researcher at Distributed Interactive Systems group of The Dutch National Research Institute for Mathematics and Computer Science (CWI). She holds a $\mathrm{PhD}$ degree in Human Information communication Design from Delft University of Technology, and is specialised in UX and HCI research. She is currently working on develop subjective metrics for assessing experience in social VR, building and evaluating new social VR experiences in diverse domains (e.g., medical consultation, co-design, museum).

Vinoba Vinayagamoorthy is a Project Research and Development Engineer at the BBC. She has research interests in $\mathrm{HCI}$, VR, AR, connected TV, user research, mobile devices, device synchronisation, interaction design and serious games. Her work has appeared in conference and journal publications including ACM SIGCHI, ACM IMX (TVX), ACM VRST, Eurographics, IVA, CGF and IEEE TVCG. She has organized workshops, served on program committees, is ACM IMX VP for Conferences and a SIGCHI Adjunct Chair for Equity.

Julie Williamson is a lecturer in human computer interaction in the School of Computing Science at the University of Glasgow. Her research focuses on interaction in public spaces, including non-planar displays, virtual reality, and tangible interfaces. She is an expert in public evaluation and research on social acceptability of novel technologies.

David Ayman Shamma is an industry research scientist, adjunct faculty at Rochester Institute of Technology, and SIGCHI VP of Operations. Recently he was a senior research scientist at FX Palo Alto Labratory (FXPAL). Prior to FXPAL, he was a principal investigator at Centrum Wiskunde \& Informatica (CWI) where he lead a project on Artificial Intelligence (AI), wearables, and fashion. Before CWI, he was the founding director of the HCI Research Group at Yahoo Labs and Flickr. He investigates social computing systems (how people interact, engage, and share media experiences both online and in-the-world) through three avenues: AI, systems \& prototypes, and qualitative research; his goal is to create and understand methods for media-mediated communication in small environments and at web scale.

Pablo Cesar leads Distributed and Interactive Systems group at CWI (The Dutch National Research Institute for Mathematics and Computer Science) and is a professor of human-centered multimedia computing at the Delft University of Technology. Pablo's research focuses on modeling and controlling complex collections of media objects (including real-time media and sensor data) that are distributed in time and space. Pablo has given tutorials about multimedia systems in prestigious conferences such as ACM Multimedia, CHI, and the WWW conference. He acted as an invited expert at the European Commission's Future Media Internet Architecture Think Tank and participates in standardization activities at MPEG (point-cloud compression) and ITU (QoE for multi-party tele-meetings). 


\section{ACKNOWLEDGMENTS}

This workshop proposal is partly funded by the European Commission as part of the H2020 program, under the grant agreement 762111, "VRTogether" (http://vrtogether.eu/).

\section{REFERENCES}

[1] Mark S. Ackerman, Jack Muramatsu, and David W. McDonald. 2010. Social regulation in an online game: Uncovering the problematics of code. Proceedings of the 16th ACM International Conference on Supporting Group Work, GROUP'10 (2010), 173-182. https://doi.org/10.1145/1880071.1880101

[2] John G Apostolopoulos, Philip A Chou, Bruce Culbertson, Ton Kalker, Mitchell D Trott, and Susie Wee. 2012. The road to immersive communication. Proc. IEEE 100, 4 (2012), 974-990.

[3] Michael Argyle. 1988. Bodily Communication. Routledge.

[4] Michael Argyle and Janet Dean. 1965. Eye-Contact, Distance and Affiliation. Sociometry 28, 3 (1965), 289-304. http://www.jstor.org/stable/2786027

[5] Michael Argyle and Peter Trower. 1979. Person to person: ways of communicating. Harper and Row.

[6] Andrea Bonsch, Sina Radke, Heiko Overath, Laura M. Asche, Jonathan Wendt, Tom Vierjahn, Ute Habel, and Torsten W. Kuhlen. 2018. Social VR: How Personal Space is Affected by Virtual Agents' Emotions. 25th IEEE Conference on Virtual Reality and 3D User Interfaces, VR 2018 - Proceedings (2018), 199-206. https: //doi.org/10.1109/VR.2018.8446480

[7] Jed R Brubaker, Gina Venolia, and John C Tang. 2012. Focusing on shared experiences: moving beyond the camera in video communication. In Proceedings of the Designing Interactive Systems Conference. ACM, ACM, 96-105.

[8] Minghao Cai and Jiro Tanaka. 2019. Go together: providing nonverbal awareness cues to enhance co-located sensation in remote communication. Human-centric Computing and Information Sciences 9, 1 (2019), 19.

[9] Edward Hall. 1969. The Hidden Dimension : man's use of space in public and in private. 217 pages.

[10] Leslie A. Hayduk. 1983. Personal space: Where we now stand. Psychological Bulletin 94, 2 (1983), 293-335. https://doi.org/10.1037//0033-2909.94.2.293

[11] Heiko Hecht, Robin Welsch, Jana Viehoff, and Matthew R. Longo. 2019. The shape of personal space. Acta Psychologica 193, April 2018 (2019), 113-122. https://doi.org/10.1016/j.actpsy.2018.12.009

[12] Kori Inkpen, Brett Taylor, Sasa Junuzovic, John Tang, and Gina Venolia. 2013 Experiences2Go: sharing kids' activities outside the home with remote family members. In Proceedings of the 2013 CSCW. ACM, 1329-1340.

[13] Jie Li, Vinoba Vinayagamoorthy, Raz Schwartz, Wijnand IJsselsteijn, David A Shamma, and Pablo Cesar. 2020. Social VR: A New Medium for Remote Communication and Collaboration. In Extended Abstracts of the $2020 \mathrm{CHI}$ Conference on
Human Factors in Computing Systems. ACM, 1-8.

[14] Joshua McVeigh-Schultz, Anya Kolesnichenko, and Katherine Isbister. 2019. Shaping Pro-Social Interaction in VR. (2019), 1-12. https://doi.org/10.1145/3290605. 3300794

[15] Robert Moore, E. Hankinson Gathman, and Nicolas Ducheneaut. 2009. From 3D space to third place: The social life of small virtual spaces. Human Organization 68, 2 (2009), 230-240. https://doi.org/10.17730/humo.68.2.q673k16185u68v15

[16] Fares Moustafa and Anthony Steed. 2018. A longitudinal study of small group interaction in social virtual reality. In Proceedings of the ACM Symposium on Virtual Reality Software and Technology, VRST, Vol. 10. Association for Computing Machinery, New York, NY, USA, 1-10. https://doi.org/10.1145/3281505.3281527

[17] Joschka Mütterlein, Sebastian Jelsch, and Thomas Hess. 2018. Specifics of Collaboration in Virtual Reality: How Immersion Drives the Intention to Collaborate.. In PACIS. 318.

[18] Fiachra O'Brolcháin, Tim Jacquemard, David Monaghan, Noel O'Connor, Peter Novitzky, and Bert Gordijn. 2016. The convergence of virtual reality and social networks: threats to privacy and autonomy. Science and engineering ethics 22,1 (2016), 1-29.

[19] Catlin Pidel and Philipp Ackermann. 2020. Collaboration in Virtual and Augmented Reality: A Systematic Overview. Springer, Cham, 141-156. https: //doi.org/10.1007/978-3-030-58465-8_10

[20] Albert E. Scheflen and Alice Scheflen. 1972. Body Language and the Social Order: Communication As Behavioral Control.

[21] Harrison Jesse Smith and Michael Neff. 2018. Communication behavior in embodied Virtual Reality. In Proceedings of the ACM CHI2018. ACM, 289.

[22] Duck Steve. 1998. Human Relationships.

[23] Tim Szigeti, Kevin McMenamy, Roland Saville, and Alan Glowacki. 2009. Cisco telepresence fundamentals. Cisco Press.

[24] Shari M. Trewin, Mark R. Laff, Anna C. Cavender, and Vicki L. Hanson. 2008. Accessibility in virtual worlds. Conference on Human Factors in Computing Systems - Proceedings (2008), 2727-2732. https://doi.org/10.1145/1358628.1358752

[25] Vinoba Vinayagamoorthy, Maxine Glancy, Christoph Ziegler, and Richard Schäffer. 2019. Personalising the TV Experience using Augmented Reality: An Exploratory Study on Delivering Synchronised Sign Language Interpretation. In Proceedings of the ACM CHI 2019 Conference on Human Factors in Computing Systems. ACM, 532

[26] Nick Yee, Jeremy N. Bailenson, Mark Urbanek, Francis Chang, and Dan Merget. 2007. The unbearable likeness of being digital: The persistence of nonverbal social norms in online virtual environments. Cyberpsychology and Behavior 10, 1 (2 2007), 115-121. https://doi.org/10.1089/cpb.2006.9984

[27] Yajie Zhao, Oingguo Xu, Weikai Chen, Chao Du, Jun Xing, Xinyu Huang, and Ruigang Yang. 2019. Mask-off: Synthesizing face images in the presence of head-mounted displays. In 2019 IEEE Conference on Virtual Reality and 3D User Interfaces (VR). IEEE, 267-276. 